\title{
Long-term toxicity and survival outcomes after stereotactic ablative radiotherapy for patients with centrally located thoracic tumors
}

\author{
Banu Atalar ${ }^{1,2}$, Teuta Zoto Mustafayev², Terence T. Sio ${ }^{3}$, Bilgehan Sahin ${ }^{1}$, Gorkem Gungor ${ }^{2}$, \\ Gokhan Aydın², Bulent Yapici², Enis Ozyar ${ }^{1,2}$
}

\author{
' Department of Radiation Oncology, Acibadem MAA University, Istanbul, Turkey \\ 2 Department of Radiation Oncology, Acibadem Maslak Hospital, Istanbul, Turkey \\ ${ }^{3}$ Department of Radiation Oncology, Mayo Clinic Hospital, Phoenix, Arizona
}

Radiol Oncol 2020; 54(4): 480-487.

Received 2 April 2020

Accepted 30 May 2020

Correspondence to: Banu Atalar, M.D., Department of Radiation Oncology, Acibadem MAA University, Istanbul, Turkey. E-mail: banu.atalar@acibadem.edu.tr

Disclosure: Terence T. Sio, M.D., M.Sc., provides strategic and scientific recommendations as a member of the Advisory Board and speaker for Novocure, Inc. This position has no relation to this manuscript. All other authors have no additional conflict of interest to disclose.

Background. Stereotactic ablative radiotherapy (SABR) is effective for thoracic cancer and metastases; however, adverse effects are greater for central tumors. We evaluated factors affecting outcomes and toxicities after SABR for patients with primary lung and oligometastatic tumors.

Patients and methods. We retrospectively identified consecutive patients with centrally located lung tumors that were treated at our hospital from 2009-2016. The effects of patient, disease, and treatment-related parameters on local control (LC), overall survival (OS), and toxicity-free survival (TFS) were evaluated with multivariate analyses.

Results. Among 65 consecutive patients identified with 70 centrally located tumors, 20 tumors (28\%) were reirradiated. Median (range) total dose for all tumors was 55 (30-60) Gy in 5 (3-10) fractions. Radiographic complete response was obtained in 43 lesions (61\%). None of the analyzed factors were correlated with complete response. After a median follow-up of $57(95 \% \mathrm{Cl}$, 48-65) months, 10 tumors (14\%) relapsed and 37 patients (57\%) died; the actuarial 2-and 5 -year OS rates were $52 \%$ and $28 \%$, respectively. Median OS was significantly lower in patients with grade 3 or higher toxicity vs. lower toxicity ( 5 vs. 39 months; $P<0.001$ ). Among 17 severe toxicities, 5 were grade 5 , and 3 of them were reirradiated to the same field. Grade 3 to 5 TFS was lower with vs. without reirradiation (2-year TFS, 63\% vs. 96\%; $P=0.02$ ). Conclusions. Our study showed that modern SABR is effective for central lung tumors, and toxicities are acceptable. SABR for reirradiated central lung lesions and possibly for lesions abutting the tracheobronchial tree may result in higher risk of serious toxicities.

Key words: Iung cancer; radiation; stereotactic ablative radiotherapy; stereotactic body radiation therapy; survival outcomes; toxicity

\section{Introduction}

Because local control (LC) and survival have shown limited improvement after conventionally fractionated radiotherapy for early inoperable lung tumors, interest in alternative, hypofractionated treatment schedules has increased. Stereotactic ablative radiotherapy (SABR) has been effective for primary lung tumors, as well as pulmonary metastases that are associated with other primary organs. ${ }^{1,2}$ In early studies, biological effective doses (BEDs) to the tumor with an alpha/beta ratio of 10 $\left(\mathrm{BED}_{10}\right)$ greater than $100 \mathrm{~Gy}$ given in 3 or 4 fractions resulted in better LC and improved overall survival (OS) compared with conventional radiotherapy. ${ }^{3-5}$ However, this potential therapeutic gain can come with a risk of increased toxicities including fatal events, although they are usually rare. ${ }^{6}$ Proximity 
to the trachea or main bronchi, within $1-2 \mathrm{~cm}$ of the tracheobronchial tree (TBT), is directly related to increased toxicities observed clinically. ${ }^{6-8}$ As a result, highly fractionated ablative schedules such as 54 Gy in 3 fractions should not be used for centrally located thoracic tumors with such proximity.

Recently, the highly anticipated NRG Oncology/ Radiation Therapy Oncology Group (RTOG) 0813 trial was published. ${ }^{9}$ The maximally tolerated dose of 12 Gy per fraction over 5 fractions was reached in the study; however, the dose-limiting toxicity rate of $7.2 \%$ still gives certain clinicians pause for using a 5-fraction regimen, especially for "ultracentral" lesions. ${ }^{10-13}$ A more fractionated dosing scheme and strict adherence to the organs-at-risk constraints may still need to be defined, especially for tumors that directly invade critical structures. A phase II prospective study (LungTech) by the European Organisation for Research and Treatment of Cancer using 60 Gy in 8 fractions for central lung tumors is ongoing; another Canadian study, SUNSET, mainly focuses on ultracentral lesions using SABR techniques. ${ }^{14,15}$

With the full results of these prospective trials still unavailable, we aimed to clarify the effects of current treatment regimens and predisposing factors for increased toxicities in central lung cancers. In the current study, we identified patients treated in our center and reviewed their long-term outcomes regarding LC, OS, and toxicities after SABR for centrally located primary lung and oligometastatic tumors.

\section{Patients and methods}

\section{Patient selection and grouping}

After approval by our institutional review board, we retrospectively searched our patient database for the records of all consecutive patients treated with their first SABR course to one or more centrally located lung lesions between October 2009 and April 2016 at our hospital. Primary stage I or II non-small cell lung cancers (NSCLCs), recurrent tumors after previous irradiation (regularly fractionated treatments), and oligometastatic tumors from other primary organs were included. Lesions were grouped according to distance from the tracheobronchial tree and mediastinum: 1) tumors with gross tumor volume (GTV) and/or planning target volume (PTV) very close to or abutting the tracheobronchial tree $(\leq 1 \mathrm{~cm}) ; 2)$ tumors with GTV and/or PTV 1 to $2 \mathrm{~cm}$ away from the tracheobronchial tree; 3 ) tumors intersecting the mediastinum; and 4) tumors abutting the aorta. Patients with at least 3 months of follow-up, or patients who died within 3 months after SABR completion, were included in all of the analyses.

\section{SABR treatments}

All patients were simulated in the supine position using a wing board. Patients had 1 of 3 motion management methods: 4-dimensional computed tomography (CT) using a Respiratory Gating System (Anzai Medical) or a Real-time Position Management System (Varian Medical Systems), CT performed during 3 phases (free breathing, end-expiratory phase, and inspiratory phase), or planning CT during free-breathing or during breath-hold. CT slice thickness was set at 1 to $1.5 \mathrm{~mm}$. Positron emission tomography (PET)/CT fusion was used to assist delineation for some tumors. The target tumor (as GTV) was delineated on the maximum intensity projection when applicable or by using volumes from all 3 phases of breathing, which were united to form the internal target volume. No additional expansion was given to form the clinical target volume (i.e., clinical target volume equaled GTV). PTV margin was given as a $0.5 \mathrm{~cm}$ isotropic expansion to the internal target volume for all cases.

All patients were treated using a linear accelerator (Trilogy or TrueBeam STx; Varian Medical Systems). One patient had a tumor treated by CyberKnife (Accuray, Inc).

Organs-at-risk dose constraints and PTV coverage were done according to the RTOG study protocols. Kilovoltage portal imaging and cone beam CT were used in every fraction for every patient's treatments during the daily setup. For the patient treated by CyberKnife, the Xsight lung tracking and Synchrony systems (Accuray, Inc) were used.

Treatment dose and fractionation were determined at the discretion of the treating physician, but lower doses or more protracted schedules, in general, were used for patients undergoing reirradiation and for tumors abutting the tracheobronchial tree. BED calculations, based on alpha/beta ratios of 10 (acute) and 3 (late) evaluations, were performed conventionally on the basis of classic radiobiology principles in radiation oncology.

\section{Statistical methods and outcomes}

Toxicity-free survival (TFS) and local relapsefree survival (LRFS) were calculated as time since the end of SABR to event occurrence (death or a 
TABLE 1. Patient, tumor, and treatment characteristics for 65 patients (70 tumors) receiving stereotactic ablative radiotherapy (SABR)

\begin{tabular}{|c|c|}
\hline Characteristic & Value $^{a}$ \\
\hline Age, year & $64(22-95)$ \\
\hline Men & $50(77)$ \\
\hline \multicolumn{2}{|l|}{ Primary cancer } \\
\hline Lung & $49(70)$ \\
\hline Colorectal & $10(14)$ \\
\hline Other (breast, gastric, melanoma, germ cell, RCC) & $11(16)$ \\
\hline \multicolumn{2}{|l|}{ Treatment indication } \\
\hline Primary lung (medically inoperable T1-T2) & $12(17)$ \\
\hline Relapse (primary lung and oligometastatic) & $24(34)$ \\
\hline Oligometastatic & $34(49)$ \\
\hline Previous radiation to chest & $20(29)$ \\
\hline \multicolumn{2}{|l|}{ Tumor location } \\
\hline$\leq 1 \mathrm{~cm}$ from tracheobronchial tree & $24(34)$ \\
\hline$>1 \mathrm{~cm}$ but $\leq 2 \mathrm{~cm}$ from tracheobronchial tree & $12(17)$ \\
\hline Lesions intersecting mediastinum & $22(31)$ \\
\hline$\leq 1 \mathrm{~cm}$ from thoracic aorta & $12(17)$ \\
\hline Left laterality & $37(53)$ \\
\hline Lesion size (PTV), CC & $33.4(7.3-461.5)$ \\
\hline Total dose, Gy & $55(30-60)$ \\
\hline Dose per fraction, Gy & $9.75(4-18)$ \\
\hline Fractions & $5(3-10)$ \\
\hline $\mathrm{BED}_{10}, \mathrm{~Gy}$ & $110(48-151.2)$ \\
\hline \multicolumn{2}{|l|}{$\mathrm{BED}_{10}$} \\
\hline$<100 \mathrm{~Gy}$ & $16(23)$ \\
\hline$\geq 100 \mathrm{~Gy}$ & $54(77)$ \\
\hline $\mathrm{BED}_{3}, \mathrm{~Gy}$ & $228(90-378)$ \\
\hline Treatment time, days & $10(5-19)$ \\
\hline \multicolumn{2}{|l|}{ Treatment time } \\
\hline$<10$ days & $30(43)$ \\
\hline$\geq 10$ days & $40(57)$ \\
\hline Treatment on consecutive days & $6(9)$ \\
\hline
\end{tabular}

$\mathrm{BED}=$ biological effective dose; PTV = planned tumor volume; RCC = renal cell carcinoma; a Values are median (range) or No. of patients/tumors (\%).

grade 2 or higher toxicity for TFS and death or locoregional relapse for LRFS, whichever occurred earlier). OS for patients with multiple SABR treatments was calculated as time since the end of the last SABR to death. Toxicity was graded according to the Common Terminology Criteria for Adverse Events, 4th edition.
OS, TFS, and LRFS were calculated using the Kaplan-Meier method, and log-rank tests were used for comparison between groups. Complete response was defined as shrinkage or radiographic disappearance of the tumor on 3-month follow-up scans, with decreasing maximum standardized uptake values (SUV). Partial response was defined as minimal decrease in tumor size or maximum SUV. Progression was defined as an increase in tumor size and also maximum SUV, concerning for residual tumor or recurrence. Multivariate hazard ratios (HRs) and corresponding 95\% CIs were calculated by Cox regression analysis. Statistical analysis was performed with IBM SPSS Statistics software version 23 (IBM SPSS Statistics). All $P$ values were 2-sided, and $P<0.05$ was considered statistically significant.

\section{Results}

Our search identified 65 patients (70 lesions) with at least 3 months of follow-up or who died within 3 months after SABR completion. The type of tumor was primary lung in $49(70 \%)$ and oligometastatic in 21 (30\%). The patient, tumor, and treatment characteristics are summarized in Table 1. The treatment planning was 4-dimensional CT in 15 patients (23\%), CT during 3 phases in $43(66 \%)$, and CT during free-breathing or during breathhold in 7 (11\%). PET/CT fusion was used to assist delineation for 50 patients (77\%). Volumetric modulated arc therapy was the most commonly used technique $(34,52 \%)$, followed by 3-dimensional conformal $(29,45 \%)$ and dynamic conformal arc (2, $3 \%$ ) radiotherapies. Median (range) total dose was 55 Gy (30-60 Gy), fraction dose was 9.75 Gy (4-18 Gy), BED 10 was 110 Gy (41-151 Gy), and $\mathrm{BED}_{3}$ was 228 Gy (90-378 Gy). The median (range) number of fractions was 5 (3-10).

Reirradiation was performed for 20 tumors $(28 \%)$ (Table 1). The median dose given as reirradiation was lower than for other tumors (reirradiation $\mathrm{BED}_{10}$ dose: $94.4 \mathrm{~Gy}$ reirradiation vs. $110 \mathrm{~Gy}$ non-reirradiation; $P=0.009$ ).

After a median follow-up of 57 months $(95 \% \mathrm{CI}$, 48-65 months), 43 (61\%) of the tumors achieved complete response (Table 2). On univariate analysis, $\mathrm{BED}_{10}(>100$ vs. $\leq 100 \mathrm{~Gy})$, PTV size (> 33.4 vs. $\leq 33.4 \mathrm{cc}$ ), and type of tumor (colorectal metastases vs. other tumors) were not related to complete response radiographically by $\mathrm{PET} / \mathrm{CT}$ at 3 months after the end of SABR treatments (all $P>0.05$ ). 


\section{Locoregional control and survival}

LRFS was lower in patients with colorectal cancer as a primary tumor (2-year LRFS: colorectal metastases, $59 \%$ vs. other primary tumors, $89 \% ; P=$ 0.02) (Figure 1A). LRFS also was lower in tumors that did not have a complete response 3 months after the end of SABR (2-year LRFS: no complete response, $51 \%$ vs. complete response, $100 \% ; P<$ 0.001) (Figure 1B). On multivariate analyses, tumors with less than complete response had lower LRFS (HR, 18.2; 95\% CI, 2.3-145.9; $P=0.006$ ). Other factors, including previous radiotherapy, $\mathrm{BED}_{10}$ greater than $100 \mathrm{~Gy}$, PTV size, or tumor location in relation to the tracheobronchial tree, had no effect on local relapse (all $P>0.05$ ).

\section{Overall survival}

During follow-up, 10 tumors (14\%) relapsed (2and 5-year LC were $84 \%$ and $70 \%$, respectively), and 37 patients $(57 \%)$ died (2- and 5-year OS were $52 \%$ and $28 \%$, respectively). Median OS was significantly lower in patients who had toxicity of grade 3 or higher ( 5 months, grade $\geq 3$ toxicity vs. 39 months grade $<3$ toxicity) (Figure 2A). Grade 3 or higher toxicity conferred a significantly increased risk of death (HR, 4.7, 95\% CI, 2.0-11.2; $P<0.001)$. Median OS was slightly lower in patients with primary lung cancer than in patients with other primary cancer origins (19 months, lung cancer vs. 49 months, other cancers) (Figure 2B), but the risk of death was not significantly increased (HR, 2.3; 95\% CI, 1.0-5.6; $P=0.06$ ). Factors including previous radiotherapy, $\mathrm{BED}_{10}$ higher than $100 \mathrm{~Gy}$, PTV size, or position of the lesions in relation to the tracheobronchial tree had no effect on OS (all $P>0.05$ ).

\section{SABR-related toxicities}

Seventeen toxicities of grade 2 or higher were observed in 13 patients, some patients have more than 1 toxicity (Table 2). Imaging examples of patients with tracheal rupture and vocal cord paralysis are shown in Figure 3. The most common toxicity was radiation-induced pneumonia. Less common toxicities, including brachial plexus injury (giving rise to Lhermitte sign) and vocal cord paralysis (due to vagus or recurrent laryngeal nerve injury), were observed in 3 patients; radiation-related esophagitis occurred in 2 patients.

Seven of the 10 toxicities of grade 3 to 5 were observed in reirradiation patients, which conferred an HR of 5.8 (95\% CI, 1.7-20.3). Also, 7 of 10 grade
TABLE 2. Tumor and patient outcomes after stereotactic ablative radiotherapy (SABR) for central lung tumors

\begin{tabular}{lc}
\hline Characteristic & Value $^{\mathrm{a}}$ \\
\hline Response on 3-month PET/CT after SABR & \\
$\quad$ Complete response & $43(61)$ \\
Partial response & $19(27)$ \\
Progression & $2(3)$ \\
Unknown (patient died before 3 months or imaging not & $6(9)$ \\
performed) & \\
Locoregional control & $84 \%$ \\
2-year & $70 \%$ \\
5-year & Not reached \\
Median & \\
Overall survival & $52 \%$ \\
2-year & $28 \%$ \\
5-year & $28 \mathrm{months}$ \\
Median & $81 \%$ \\
2-Year toxicity-free survival & $17(26.2 \%)$ \\
All Toxicities (grade 2 or higher) & $9(13.8 \%)$ \\
RT-induced pneumonitis & $3(4.6 \%)$ \\
Brachial and recurrent laryngeal nerve injury & $2(3 \%)$ \\
Esophagitis & $1(1.5 \%)$ \\
Tracheal perforation & $1(1.5 \%)$ \\
Fatal hemoptysis & $1(1.5 \%)$ \\
Possible RT-related death & $5(7.7 \%)$ \\
\hline
\end{tabular}

$\mathrm{PET} / \mathrm{CT}$ = positron emission tomography/computed tomography; RT = radiotherapy: a Values are No. patients/tumors (\%) or No. patients unless otherwise stated.
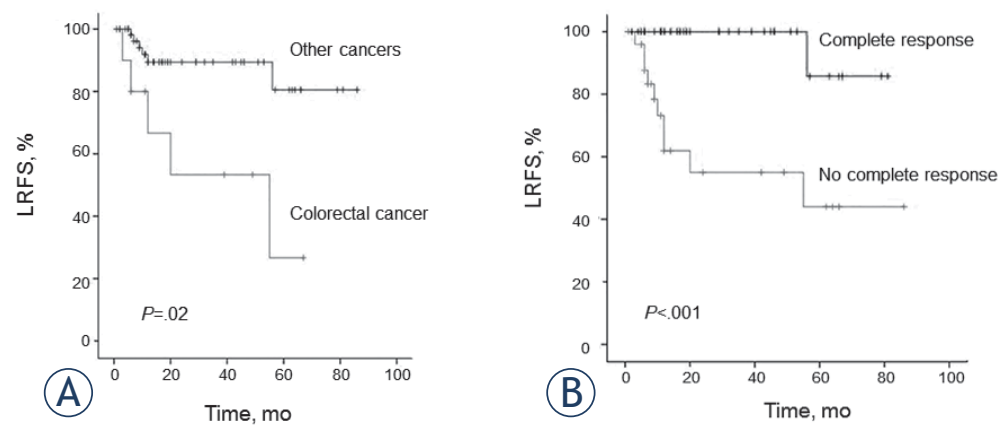

FIGURE 1. Kaplan-Meier curves for locoregional relapse-free survival (LRFS). (A) LRFS of all patients according to primary tumor type (colorectal cancer vs. others). (B) LRFS of all patients according to radiographic response 3 months after radiotherapy (complete response vs. no complete response). Tick marks on lines indicate censored patients. 

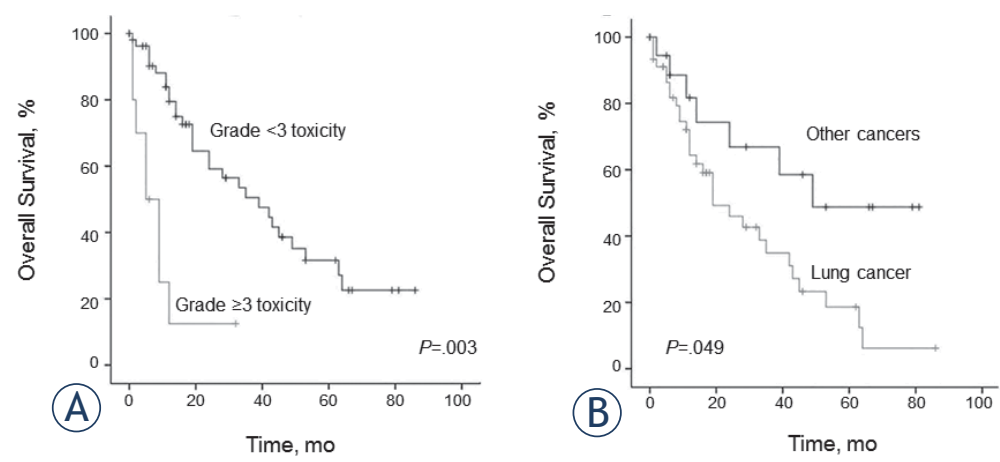

FIGURE 2. Kaplan-Meier curves for overall survival (OS). (A) OS of all patients according to development of a grade 3 or higher toxicity (vs. not). (B) OS of all patients according to primary tumor type (lung cancer vs. others). Tick marks on lines indicate censored patients.

3 to 5 toxicities were observed in lesions abutting the tracheobronchial tree, for an HR of $4.5(95 \% \mathrm{CI}$, 1.3-15.8). Among the 17 toxicities, 5 were grade 5 (fatal). Three out of 5 fatal toxicity patients were reirradiated to the same RT field, and one of them was irradiated to a neighboring field. The prior and reirradiation doses of each patients were 66Gy/33 fractions and $30 \mathrm{~Gy} / 5$ fractions; $40 \mathrm{~Gy} / 10$ fractions and $59.5 \mathrm{~Gy} / 7$ fractions; $66 \mathrm{~Gy} / 33$ fractions and 30 Gy/5 fractions; and 45 Gy/15 fractions with the neighboring field dose and $50 \mathrm{~Gy} / 5$ fractions, respectively. We were able to get the medical reports and the thoracic CT for 3 of the patients and confirmed the grade 5 toxicity; in regard to patient \#4, which was reported as "possible RT-related death," this was due to the fact that his death was unexpected, and happened only a few weeks shortly after his SABR course; this information was given to us by his relatives. To be estimating this toxicity rate conservatively, we believe that it is reasonable to account for this in the statistics (so it did not appear that we were biased), as the death did happen within one month after SABR. The last patient who had grade 5 toxicity after 1st SABR was treated to a totaldose of 59.5Gy in 7 fractions and notably he had a lesion encasing bronchus with a size of 55 $\mathrm{mm}$ which was considered to be a larger lesion for SABR. After a reasonable amount of effort, we could not locate his radiological images; however, the emergency medical notes noted symptoms and signs of him developing an acute pneumonia. As a result, we considered the possibility that it could be a RT-related pneumonia due to the proximity of timing to his SABR course.

Survival free of grade 3 to 5 toxicity was lower after reirradiation than in patients without reirradiation (2-year TFS: 63\% after reirradiation vs. 96\% without reirradiation) (Figure 4A); the HR was 5.1 (95\% CI, 1.3-20.3; $P=0.02$ ). TFS also was lower in tumors abutting the tracheobronchial tree (2-year TFS: $69 \%$, tumors abutting the tracheobronchial tree vs. 93\%, other cases) (Figure 4B), but the associated risk did not reach statistical significance (HR, 3.5; 95\% CI, 0.9-13.9; $P=0.08$ ).

\section{Discussion}

Grade 3 or higher complications of SABR for centrally located lung tumors are still a substantial concern, as reported by multiple studies, including the most recently published NRG Oncology/ RTOG 0813 trial. 5,6,8,9,12 Therefore, more studies are required to evaluate whether these findings are similar in the general population. To our knowledge, the current retrospective study is one of the
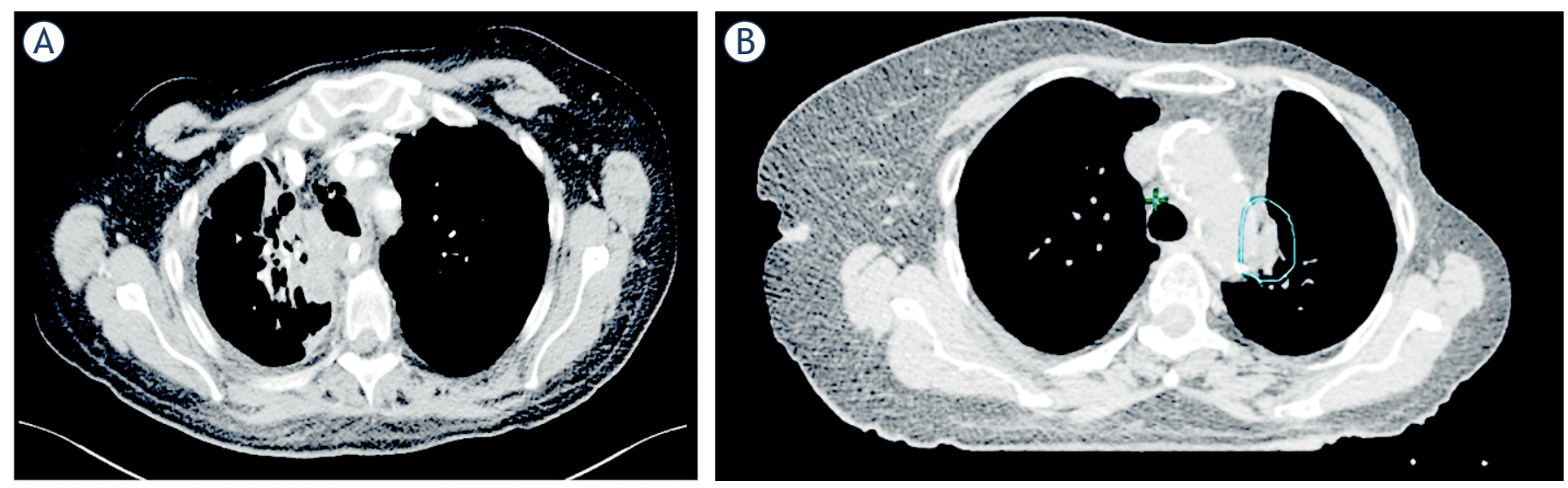

FIGURE 3. Computed tomographic imaging examples of patients with a grade 3 or higher toxicity. (A) Patient with a tracheal rupture after reirradiation. (B) Patient with vocal cord paralysis after reirradiation (previous chest wall radiotherapy). The circled portion indicates the planning target volume. 
largest series to date for centrally located and ultracentral lung tumors. Favorable outcome and toxicity profiles were achieved, which supports the use of 5-fraction and also moderately hypofractionated regimens in this population.

The LC rates in our series are comparable to those of other published series which showed excellent tumor control. Although we saw no correlation of $\mathrm{BED}_{10}$ doses higher than $100 \mathrm{~Gy}$ with better LC, previous studies indicated that $\mathrm{BED}_{10}$ of 100 Gy or higher led to better local progressionfree survival and OS.,3 The reason for the lack of correlation in our study may be the high number of reirradiation lesions, which were prescribed lower radiotherapy doses (mean reirradiation $\mathrm{BED}_{10}$ dose, $94.4 \mathrm{~Gy}$ ). reirradiation lesions also had shorter follow-up, so their local recurrence rates may appear lower at the time of data analysis. The difference also may relate to the heterogeneity of these tumors, including colorectal oligometastatic, lung cancers with epidermal growth factor receptor or anaplastic large-cell lymphoma kinase-gene mutations, and other confounding factors such as chemotherapy before or after SABR. If only nonreirradiation primary lung lesions are considered, the LC rates in our study (2-year LC, 71\%) are similar to those in the literature. ${ }^{2}$ Metastatic tumors with a separate primary seemed to have higher LC rates (2-year LC, $81 \%$ ) than those reported in the literature $(51 \%-96 \%$, with various radiotherapy doses). ${ }^{1}$ At this time, there is no clear correlation between LC and radiotherapy doses, although LC was found to be positively correlated with favorable response radiographically 3 months after SABR by PET/CT in our study (the use of PET/CT for follow-up is a routine practice at our institution).

In our series, 2- and 5-year OS were $48 \%$ and $20 \%$, respectively, for patients with primary lung cancer and were $60 \%$ and $44 \%$, respectively, for patients with oligometastatic tumors. The 2-year OS rates in the literature range from $33 \%$ to $84 \%$ depending on primary tumor type, size and number of lesions, disease-free survival from primary tumor treatment to onset of metastasis, and other treatment-related factors. ${ }^{1}$ Similarly, survival after SABR for patients with NSCLC has also varied among studies, with 2-year OS ranging from $43 \%$ to $90 \%$ depending on radiotherapy dose, tumor size, clinical performance status, and tumor location (central vs. not). ${ }^{2}$ With $29 \%$ of our tumor cases being reirradiation and $16 \%$ of tumors being larger than $5 \mathrm{~cm}$, our results are comparable to the historical controls as a result. The higher rates of toxicities
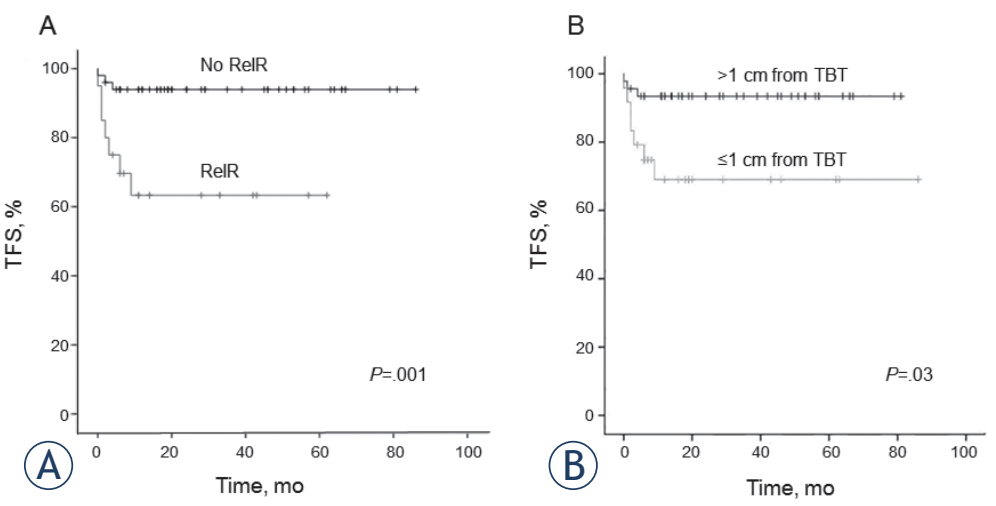

FIGURE 4. Kaplan-Meier Curves for grade 3 or higher toxicity-free survival (TFS). (A) TFS for all patients according to reirradiation vs. no reirradiation. (B) TFS for all patients according to the distance of the planning target volume from the tracheobronchial tree (> $1 \mathrm{~cm}$ or $\leq 1 \mathrm{~cm}$ ). Tick marks on lines indicate censored patients.

(including grade 5 cases) also contributed to the lower OS rates in our study.

Compared with rates reported in the literature, a slightly higher rate of possible grade 5 toxicities was noted in our cohort; 5 patients who died had treatment complications that may have been causative, including pneumonitis, tracheal perforation, and hemoptysis. OS in patients with grade 3 to 5 toxicity was short, with a median of only 5 months after SABR. Reirradiation carried significant risks in these cases because it resulted in a high cumulative dose in the mediastinum. More guidance and research in the future are required for making SABR safer in these clinical scenarios, in which patients often have no other choice but reirradiation, along with proper counseling regarding potential treatment outcomes and adverse effects.

For centrally located lung tumors or nodal recurrences after previous irradiation, some authors have discouraged the use of SABR because of the perceived high risks of toxicity. ${ }^{16,17}$ In other studies that included central lesions without prior radiotherapy, a higher rate of grade 5 toxicities was often reported. ${ }^{16-18}$ In an analysis of 32 lesions (11 central) that were previously irradiated, Peulen et al. reported that treatment of central lung lesions and lesions with larger volumes resulted in higher toxicity; 9 of 29 patients had grade 3 or higher toxicity, including 3 cases of fatal hemoptysis. ${ }^{17}$ Another prospective trial studying salvage SABR in NSCLC did not include any central lesions in their reirradiation series. ${ }^{18}$

The GTV or PTV was within $1 \mathrm{~cm}$ of the tracheobronchial tree (ultracentral) in 24 (34\%) of our patients. Four of these patients had grade 5 toxicity. Because 3 of those patients also had reirradiation, 
we do not know conclusively whether the death was related to reirradiation, tumor proximity to the tracheobronchial tree, or both. The literature reports conflicting results regarding the importance of proximity to the tracheobronchial tree (lesions abutting the tracheobronchial tree vs. other central lesions), with some studies considering these lesions as harboring similar risk as other central tumors and other studies advocating for more caution in their treatment planning. $7,8,10,13$

Vocal cord paralysis is a rarely recognized complication of SABR. To our knowledge, only 2 studies have reported its occurrence. ${ }^{19,20}$ Shultz et al. concluded that reirradiation to the vagal or recurrent laryngeal nerve in 1 case and connective tissue disorders in another case led to nerve injury and paralysis of the vocal cord. ${ }^{20}$ Two of our patients had vocal cord paralysis, which was confirmed by laryngoscopy. In both patients, PET/CT was performed at the onset of voice hoarseness to exclude local recurrence or as part of follow-up: None of the patients had lesions that would otherwise explain their symptoms. One of the patients had had SABR to the same lesion previously, and the other patient had previous ipsilateral breast irradiation (the contribution from the previous breast radiotherapy was estimated to be about $15 \mathrm{~Gy}$ to the new GTV [by SABR]). Both lesions were located adjacent to the aortic arch and invaded the vagus nerve; they were also in close proximity to the recurrent laryngeal nerve (Figure 3).

Our study has several limitations. The study was retrospective, and the patient population was more heterogeneous than in other reported series on this topic (in terms of radiotherapy dose and also inclusion of primary lung vs. oligometastatic tumors). Because our institution is a tertiary referral center, some patients' follow-up was not completed in our department. The circumstances related to patients' death were derived from interviews with relatives instead of medical records, which led us to recategorize 1 of the grade 5 toxicities as SABR related instead of "unknown cause." Heterogeneity and lower patient numbers in different subgroups also may have limited our study power.

\section{Conclusions}

SABR is an effective treatment modality for centrally located lung cancers. SABR to reirradiation lesions, and possibly lesions abutting the tracheobronchial tree, appeared to carry a higher risk of higher grade toxicities developing in the long term.
More research is needed to define the optimal dose and fractionation schedule for both centrally and ultracentrally located lung tumors. We are waiting for completion of more prospective trials, which will hopefully give more information regarding suitable treatment regimens and clearer factors that may predispose patients to increased toxicities after SABR for central lung cancers.

\section{Acknowledgment}

Portions of this manuscript were presented at the International Association for the Study of Lung Cancer 19th World Conference on Lung Cancer, Toronto, Canada, September 23-26, 2018.

\section{References}

1. Shultz DB, Filippi AR, Thariat J, Mornex F, Loo BW, Jr., Ricardi U. Stereotactic ablative radiotherapy for pulmonary oligometastases and oligometastatic lung cancer. J Thorac Oncol 2014; 9: 1426-33. doi:10.1097/ JTO.0000000000000317

2. Shah JL, Loo BW, Jr. Stereotactic ablative radiotherapy for early-stage lung cancer. Semin Radiat Oncol 2017; 27: 218-28. doi: 10.1016/j.semradonc.2017.03.001

3. Lagerwaard FJ, Haasbeek CJ, Smit EF, Slotman BJ, Senan S. Outcomes of risk-adapted fractionated stereotactic radiotherapy for stage I non-small-cell lung cancer. Int J Radiat Oncol Biol Phys 2008; 70: 685-92. doi: 10.1016/j. ijrobp.2007.10.053

4. Timmerman R, Paulus R, Galvin J, Michalski J, Straube W, Bradley J, et al. Stereotactic body radiation therapy for inoperable early stage lung cancer JAMA 2010; 303: 1070-6. doi: 10.1001/jama.2010.261

5. Onishi H, Araki T, Shirato H, Nagata Y, Hiraoka M, Gomi K, et al. Stereotactic hypofractionated high-dose irradiation for stage I nonsmall cell lung carcinoma: clinical outcomes in 245 subjects in a Japanese multiinstitutional study. Cancer 2004; 101: 1623-31. doi: 10.1002/cncr.20539

6. Timmerman R, McGarry R, Yiannoutsos C, Papiez L, Tudor K, DeLuca J, et al. Excessive toxicity when treating central tumors in a phase II study of stereotactic body radiation therapy for medically inoperable early-stage lung cancer. J Clin Oncol 2006; 24: 4833-9. doi: 10.1200/JCO.2006.07.5937

7. Tekatli H, Haasbeek N, Dahele M, De Haan, Verbakel PW, Bongers E, et al. Outcomes of hypofractionated high-dose radiotherapy in poor-risk patients with "ultracentral" non-small cell lung cancer. J Thorac Oncol 2016; 11: 1081-9. doi: $10.1016 /$ j.jtho.2016.03.008

8. Haseltine JM, Rimner A, Gelblum DY, Modh A, Rosenzweig KE, Jackson A, et al. Fatal complications after stereotactic body radiation therapy for central lung tumors abutting the proximal bronchial tree. Pract Radiat Oncol 2016; 6: e27-33. doi: 10.1016/j.prro.2015.09.012

9. Bezjak A, Paulus R, Gaspar LE, Timmerman RD, Straube WL, Ryanet WF, al. Safety and efficacy of a five-fraction stereotactic body radiotherapy schedule for centrally located non-small-cell lung cancer: NRG Oncology/RTOG 0813 Trial. J Clin Oncol 2019; 37: 1316-25. doi: 10.1200/JCO.18.00622

10. Chaudhuri AA, Tang C, Binkley MS, Jin M, Wynne JF, von Eyben R, et al. Stereotactic ablative radiotherapy (SABR) for treatment of central and ultra-central lung tumors. Lung Cancer 2015; 89: 50-6. doi: 10.1016/j.lungcan.2015.04.014

11. Chang JY, Li QQ, Xu QY, Allen PK, Rebueno N, Gomez DR, et al. Stereotactic ablative radiation therapy for centrally located early stage or isolated parenchymal recurrences of non-small cell lung cancer: how to fly in a "no fly zone". Int J Radiat Oncol Biol Phys 2014; 88: 1120-8. doi: 10.1016/j. ijrobp.2014.01.022 
12. Haasbeek CJ, Lagerwaard FJ, Slotman BJ, Senan S. Outcomes of stereotactic ablative radiotherapy for centrally located early-stage lung cancer. $J$ Thorac Oncol 2011; 6: 2036-43. 12. doi: 10.1097/JTO.0b013e31822e71d8

13. Raman S, Yau V, Pineda S, Le LW, Lau A, Bezjak A, et al. Ultracentral tumors treated with stereotactic body radiotherapy: single-institution experience. Clin Lung Cancer 2018; 19: e803-e810. doi: 10.1016/j.cllc.2018.06.001

14. Adebahr S, Collette S, Shash E, Lambrecht M, Le Pechoux C, Faivre-Finn C, et al. LungTech, an EORTC Phase II trial of stereotactic body radiotherapy for centrally located lung tumours: a clinical perspective. Br J Radiol 2015; 88: 20150036. doi: 10.1259/bjr.20150036

15. Giuliani M, Mathew AS, Bahig H, Bratman SV, Filion E, Glicket D, et al. SUNSET: stereotactic radiation for ultracentral non-small-cell lung cancer - a safety and efficacy trial. Clin Lung Cancer 2018; 19: e529-32. doi: 10.1016/j. cllc.2018.04.001

16. Matsuo Y. A systematic literature review on salvage radiotherapy for local or regional recurrence after previous stereotactic body radiotherapy for lung cancer. Technol Cancer Res Treat 2018; 17: 1533033818798633 . doi: $10.1177 / 1533033818798633$

17. Peulen $\mathrm{H}$, Karlsson K, Lindberg K, Tullgren O, Baumann P, Lax I, et al. Toxicity after reirradiation of pulmonary tumours with stereotactic body radiotherapy. Radiother Oncol 2011; 101: 260-6. doi: 10.1016/j.radonc.2011.09.012

18. Sun B, Brooks ED, Komaki R, Liao Z, Jeter M, McAleer M, et al. Long-term outcomes of salvage stereotactic ablative radiotherapy for isolated lung recurrence of non-small cell lung cancer: a phase II clinical trial. J Thorac Oncol 2017; 12: 983-92. doi: 10.1016/j.jtho.2017.02.018

19. Binkley MS, Hiniker SM, Chaudhuri A, Maxim PG, Diehn M, Loo BW Jr, et al. Dosimetric factors and toxicity in highly conformal thoracic reirradiation. Int J Radiat Oncol Biol Phys 2016; 94: 808-15. doi: 10.1016/j.jjrobp.2015.12.007

20. Shultz DB, Trakul N, Maxim PG, Diehn M, Loo BW, Jr. Vagal and recurrent laryngeal neuropathy following stereotactic ablative radiation therapy in the chest. Pract Radiat Oncol 2014; 4: 272-8. doi: 10.1016/j.prro.2013.08.005 